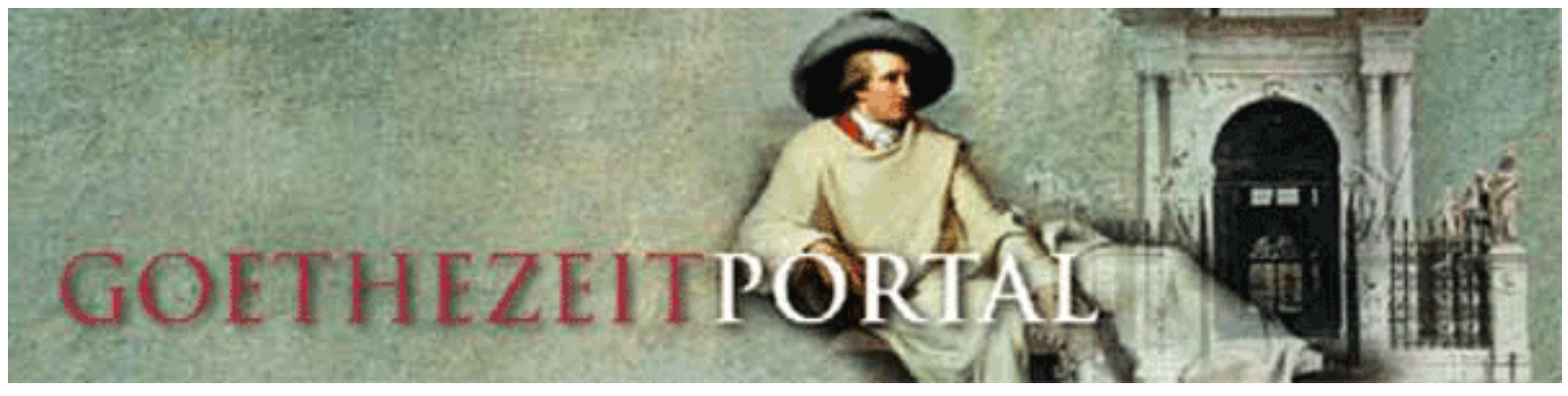

VOLKER C. DÖRR

\title{
„... bey einer guten Handlung böse Grundsätze zu argwohnen!“ \\ Empfindsame Diskurse bei Gellert, Sophie von La Roche und in Goethes Werther
}

\section{Vorblatt}

\section{Publikation}

Erstpublikation: Orbis Litterarum, hg. v. Morten Nøjgaard, Lars Ole Sauerberg und Bengt Algot Sørensen, Oxford: Blackwell Publishing, 55 (2000), S. 58-79. Neupublikation im Goethezeitportal

Vorlage: Datei des Autors

URL: <http://www.goethezeitportal.de/db/wiss/epoche/doerr_diskurse.pdf>

Eingestellt am 17.05.2004

\section{Autor}

PD Dr. Volker C. Dörr

Rheinische Friedrich-Wilhelms-Universität Bonn

Germanistisches Seminar

Am Hof 1d

53113 Bonn

Tel. + 49-(0)228-73-7425

Emailadresse: vcdoerr@uni-bonn.de

\section{Empfohlene Zitierweise}

Beim Zitieren empfehlen wir hinter den Titel das Datum der Einstellung oder des letzten Updates und nach der URL-Angabe das Datum Ihres letzten Besuchs dieser Online-Adresse anzugeben:

Volker C. Dörr: ,,... bey einer guten Handlung böse Grundsätze zu argwohnen!“ Empfindsame Diskurse bei Gellert, Sophie von La Roche und in Goethes Werther (17.05.2004). In: Goethezeitportal. URL:

$<$ http://www.goethezeitportal.de/db/wiss/epoche/doerr_diskurse.pdf> (Datum Ihres letzten Besuches). 


\title{
VOLKER C. DÖRR
}

\section{„... bey einer guten Handlung böse Grundsätze zu argwohnen!“ Empfindsame Diskurse bei Gellert, Sophie von La Roche und in Goethes Werther}

\begin{abstract}
Drei paradigmatische empfindsame Romane - Gellerts Das Leben der Schwedischen Gräfinn von $G^{* * *}$, Sophie von La Roches Geschichte des Fräuleins von Sternheim und Goethes Werther - werden darauf hin befragt, was sich ihnen hinsichtlich der diskursiven Struktur der Empfindsamkeit ablesen läßt. Dabei werden, aufbauend auf Foucaults Bestimmungen, ein starker und ein schwacher Diskursbegriff unterschieden: Kriterium der Unterscheidung ist, ob eine 'Formation' sich selbst als Diskurs organisiert oder ob sie nur (nachträglich) als Diskurs beschrieben werden kann. Mit Hilfe dieser Differenz läßt sich eine signifikante, notwendige Entwicklung der Empfindsamkeit nachweisen, die die drei Romane auf unterschiedliche Weise reflektieren.
\end{abstract}

Die Behauptung, daß sich die Empfindsamkeit „gegen die Vorherrschaft des Rationalismus in einer verinnerlichten Aufklärung unter dem Einfluß des Pietismus“ entwickelt habe und „die Verweltlichung des relig. Naturgefühls in subjektiven Gefühlsüberschwang“ bedeute ${ }^{1}$, kann heute kaum noch vollständig überzeugen. Was den Zusammenhang mit dem Pietismus angeht, so ist zwar die genetische Ableitung - auch gegen Aufforderungen zu einer polyperspektivischen Betrachtungsweise $^{2}$ - längst zum Gemeinplatz geworden; gerade als solcher aber scheint sie den Blick auf wesentliche strukturelle Merkmale der Empfindsamkeit eher zu verstellen als zu schärfen. Gemeinsamkeiten müssen dennoch konstatiert werden: Empfindsamkeit und Pietismus verfolgen ähnliche rhetorische Strategien; beide pflegen eine anti-rhetorische Rhetorik. ${ }^{3}$ Dagegen läßt sich das Verhältnis von großen Teilen der Empfindsamkeitsbewegung zur „Vorherrschaft des Rationalismus“ 'der' Aufklärung wohl weniger als Abkehr denn vielmehr als Versuch der Installation eines korrelierten Korrektivs auffassen. Dies macht exemplarisch die Lektüre zeitgenössischer theoretisch-programmatischer Texte zur Empfindsamkeit deutlich; allein solche singulären, aber nicht isolierten

\footnotetext{
1 Gero von Wilpert: Sachwörterbuch der Literatur, 7., verb. u. erw. Aufl., Stuttgart 1989, S. 231 (s. v. „Empfindsamkeit“).

2 Kritik an der einseitigen Herleitung aus dem Pietismus übt bereits: Gerhard Sauder: Empfindsamkeit, Bd. I: Voraussetzungen und Elemente; Bd. III: Quellen und Dokumente, Stuttgart 1974; 1980, hier: Bd. I, S. 58 ff.

3 Zur pietistischen Abwehr poetischer Fiktionen vgl. Sauder, Empfindsamkeit [Anm. 2], Bd. I, S. $159 \mathrm{ff}$.
} 
Begriffsungetüme wie Michael Ringeltaubes „vernunftsinnlichzärtlich“4 ${ }^{\text {zeigen, }}$ daß es um eine Vermittlungsleistung geht: um die Vermittlung der sinnlichen Natur des Menschen mit seiner Rationalität, die anders als jene zur Mitte des 18. Jahrhunderts schon eine prominente Rolle eingenommen hat. Daß die Rationalität dabei ihren zu Beginn der Empfindsamkeit fraglosen Primat schließlich vollständig verliert, macht die innere historische Entwicklung der Empfindsamkeit aus. ${ }^{5}$ 1779, zu einem Zeitpunkt also, als sich Empfindsamkeit - in welchem Sinne auch immer - weitgehend etabliert hat, grenzt Campe „wahre Empfindsamkeit“ von der bloßen „Empfindelei“ ab:

Wahre Empfindsamkeit nemlich stüzt sich immer auf deutlich erkante Grundsäze der Vernunft und harmoniert daher, sowohl mit der Natuhr des Menschen, als auch mit der Natuhr und Bestimmung anderer Dinge; Empfindelei hingegen beruhet bloos auf dunkeln Gefülen dessen, was andere Menschen für sitlich schön und für sitlich häslich halten, und steht daher nicht selten, sowohl mit der Natuhr des Menschen, als auch mit der Natuhr und Bestimmung anderer Dinge im offenbaren Widerspruche [...]. ${ }^{6}$

Nikolaus Wegmann hat vor einiger Zeit vorgeschlagen, Empfindsamkeit weniger als geistesgeschichtliches (Stichworte: Pietismus, Anti-Rationalismus) oder sozialhistorisches (Stichwort: „Reaktion e. politisch einflußlosen [...] Bürgertums““7), sondern - im Sinne Michel Foucaults - als diskursives Phänomen zu begreifen $^{8}$, und die methodischen Konsequenzen auch selbst erfolgreich umgesetzt: in einer Lektüre vor allem der zeitgenössischen theoretischen Texte. ${ }^{9}$ Dieser Weg soll hier weiter verfolgt werden.

Der Terminus 'Diskurs' erfährt bei Foucault keine trennscharfe Definition, sondern verbleibt in einer - durchaus produktiven ${ }^{10}$ - Verschwommenheit; fast typisch sind Formulierungen wie die, daß Diskurse „Ensembles diskursiver

\footnotetext{
4 Michael Ringeltaube: Von der Zärtlichkeit, Breslau/Leipzig 1765, S. 44; zitiert nach: Nikolaus Wegmann: Diskurse der Empfindsamkeit. Zur Geschichte eines Gefühls in der Literatur des 18. Jahrhunderts, Stuttgart 1988, S. 43.

5 Vgl. Georg Jäger: Empfindsamkeit und Roman. Wortgeschichte, Theorie und Kritik im 18. und frühen 19. Jahrhundert, Stuttgart/Berlin/Köln/Mainz 1969, S. 44 ff.

6 Joachim Heinrich Campe: Ueber Empfindsamkeit und Empfindelei in pädagogischer Hinsicht, Hamburg 1779, S. 13f.; zit. nach: Sauder, Empfindsamkeit [Anm. 2], Bd. III, S. 7 [Abschnitt im Original hervorgehoben]. Vgl. dazu ebd., Bd. I, S. 155.

7 Wilpert, Sachwörterbuch [Anm. 1], S. 231. - Vgl. Wolf Lepenies: Melancholie und Gesellschaft, Frankfurt a.M. 1972, S. 96ff.

8 Vgl. Nikolaus Wegmann: Zurück zur Philologie? Diskurstheorie am Beispiel einer Geschichte der Empfindsamkeit, in: Diskurstheorien und Literaturwissenschaft, hg. v. Jürgen Fohrmann und Harro Müller, Frankfurt a.M. 1988, S. 349-364.

9 Vgl. Wegmann, Diskurse der Empfindsamkeit [Anm. 4].

10 Zur Kritik an Foucault vgl. Manfred Frank: Zum Diskursbegriff bei Foucault, in: Diskurstheorien und Literaturwissenschaft [Anm. 8], S. 25-44.
} 
Ereignisse“ ${ }^{11}$ seien. Trotz und wegen der globalen Struktur der Definition durch Undefiniertes, die in solchen Bestimmungen bloß lokal konzentriert erscheint und die Foucaults Terminologie weit davon entfernt hält (und halten soll), in formallogischem Sinne eine Theorie zu bilden, ist doch weitgehend deutlich, wie der Diskursbegriff zu gebrauchen ist, wie sich eine „diskursive Praxis““12, die von Diskursen handelt, organisiert. Wesentlich ist der Aspekt der sprachlichen, zumindest zeichenhaften Organisation von Diskursen, die nichts anderes sind als diejenigen Aussagen, die - regelhaft - zu ihnen zählen: Ein Diskurs ist „eine Menge von Aussagen, die einem gleichen Formationssystem zugehören“. 13

Eine zusätzliche innere, Foucaults Begrifflichkeit zuspitzende, Differenzierung des Diskursbegriffs läßt sich aus der Frage ableiten, wer die Entscheidung hinsichtlich der Zugehörigkeit von Aussagen zu einem „Formationssystem“ oder eines sich Äußernden zur Gruppe der Teilnehmer an einem Diskurs fällt. Eine Sache ist es, eine überlieferte Menge von Aussagen dahingehend zu charakterisieren, daß sie eine faktische (möglicherweise abgestorbene) diskursive Praxis widerspiegelt - ja sie eigentlich bedeutet; eine andere Sache ist es, wenn ein Diskurs sich selbst als Diskurs begreift und organisiert, wenn er selbst seine „Verknappungsprinzipien“14 aufstellt und seine „Prozeduren der Ausschließung “ ${ }^{15}$ selbst performiert. In diesem Falle entscheidet er selbst darüber, welche Aussagen zugelassen, im logischen Sinn Elemente des Diskurses sind und welche nicht, sowie in der Folge, wer zur Partizipation am Diskurs zugelassen oder von ihm ausgeschlossen wird - etwa wegen fortgesetzter Äußerung von nichtdiskurskonformen Aussagen. Auf letztere Weise organisieren sich Kirchen, Parteien und Wissenschaften.

Es lassen sich also ein starker und ein schwacher oder - wenn man so will ein produktions- und ein rezeptionsästhetischer Diskursbegriff unterscheiden: In jenem Fall organisiert sich der Diskurs selbst und muß deswegen als faktische Organisation wahrgenommen werden; in diesem Fall läßt sich eine diskursive Praxis als Diskurs wahrnehmen - von außen und post festum. Daß sich Empfindsamkeit - mit allen ihren historischen Nuancierungen - in letzterem, schwachem Sinn durchaus produktiv als diskursive Praxis beschreiben läßt, ist hinreichend überzeugend nachgewiesen. Wie steht es aber um die womöglich historisch erfolgte Selbstorganisation?

11 Michel Foucault: Die Ordnung des Diskurses, München 1974, S. 39.

12 Michel Foucault: Archäologie des Wissens, Frankfurt a.M. 1981, S. 261.

13 Ebd., S. 156.

14 Foucault, Die Ordnung des Diskurses [Anm. 11], S. 36.

15 Ebd., S. 7. 
Zu fragen ist, ob Empfindsamkeit, genauer: ob die empfindsame Rede zur Zeit ihrer erfolgreichen Performierung (also etwa in der Zeit von 1750 bis 1780) hinreichend deutlich und zweifelsfrei von nicht-empfindsamer Rede unterschieden werden konnte und ob eventuelle „Verknappungsprinzipien“ des empfindsamen Diskurses, verstanden als Kollektivsingular, in ihm selbst reflektiert wurden.

Wenn Empfindsamkeit eine diskursive Praxis ist, die gekennzeichnet ist durch eingeforderte Wahrhaftigkeit des Ausgesagten, durch dessen gefühlsmäßige Fundierung und durch 'unmittelbare', anti-rhetorische Formen der Aussage, dann kann es fruchtbar sein, nicht nur die zeitgenössische Propaganda dieser Forderungen (als Basis möglicher „Prozeduren der Ausschließung“) einer 'archäologischen ${ }^{16}$ Lektüre zu unterziehen, sondern auch diejenige Gattung, die die Intentionen des Individuums und seine Äußerungen im Zusammenspiel mit seinem sozialen Umfeld beschreibt: den Roman. Im folgenden sollen drei prominente 'empfindsame’ Romane gelesen werden: Das Leben der Schwedischen Gräfinn von $G^{* * *}$ (1747/48) von Christian Fürchtegott Gellert, Sophie von La Roches Geschichte des Fräuleins von Sternheim (1771) und Goethes Die Leiden des jungen Werthers (1774); sie alle sollen darauf hin befragt werden, wie empfindsame Rede in ihnen produziert und rezipiert wird, wie über Intentionen kommuniziert und wie diese Kommunikation bewertet wird.

Wenn nach den Formen von Kommunikation über Intentionen im Roman gefragt werden soll, stellt sich zugleich die Frage nach der (Kommunikation über) Moral. Daher wird man im Falle Gellerts an gewichtigen Vertretern einer anderen Textsorte nicht vorbeikommen: an seinen Moralischen Vorlesungen. Auch wenn es eine illegitime Reduktion eines literarischen und deswegen polyvalenten Textes auf sein diskursives Substrat bedeutete, Gellerts Leben der Schwedischen Gräfinn von $G^{* * *}$ als bloße Umsetzung moralischer Programme im Medium des Romans ${ }^{17}$ zu lesen, so macht eine intertextuelle Lektüre, macht die Frage nach der möglichen Präsenz der Vorlesungen im Roman doch Sinn; denn es kann kein Zweifel daran bestehen, daß der Romantext die Fortsetzung eines aufklärerischen moraltheoretischen und -praktischen Diskurses mit, wenn nicht ausgeprägt poetischen, so doch narrativen Mitteln sein will (daß der Autor das offenbar auch

\footnotetext{
16 Vgl. Foucault, Archäologie des Wissens [Anm. 12], S. $191 \mathrm{ff}$.

17 Wie kalkuliert Gellert mit dem - zu seiner Zeit, 26 Jahre vor Blanckenburgs Versuch über den Roman theoretisch noch kaum behandelten - Instrument Roman operiert, hat Eckhardt MeyerKrentler gezeigt; vgl. E. M.-K.: Der andere Roman. Gellerts ‘Schwedische Gräfin’: Von der aufklärerischen Propaganda gegen den „Roman“ zur empfindsamen Erlebnisdichtung, Göppingen 1974 (Göppinger Arbeiten zur Germanistik, Bd. 128).
} 
so intendiert hat, ist noch das schwächste Argument). Gellerts Roman ist (auch) „moralische Anstalt““ ${ }^{18}$

Zwei zentrale Aspekte bestimmen die Untersuchungen der ersten Vorlesung, die Moral „nach ihrer Beschaffenheit, ihrem Umfange, und ihrem Nutzen“19 zu charakterisieren sucht. Da ist zunächst die immanente Analyse von „Beschaffenheit“ und „Umfang“ der Moral, die Deskription und Normation ebenso untrennbar verknüpft, wie sie nicht zwischen Ethik und Psychologie differenziert: Das „moralische Gut des Herzens“ stiftet deswegen völlig fraglos „höchste[s] Vergnügen“, weil „Tugend [...] die Gesundheit der Seele [ist]“. Schon im nächsten Atemzug aber wird die mögliche ganz diesseitige Verankerung der Tugend aufgehoben und diese transzendent motiviert; muß die irdische Seelengesundheit doch „zugleich der Keim der Glückseligkeit auf eine ewige Fortdauer seyn“ ${ }^{20}$ In einer geradezu paradigmatisch tautologischen Argumentation wird die moralische Hegemonie der Theologie aus dieser selbst abgeleitet: Während die Empirie lehre, daß oft ein „Unvermögen zum Guten“ hemmend wirkt, lehre die „Religion“, „daß wir dieses Verderben, dieses Unvermögen, nicht durch die bloßen Kräfte der Natur, sondern durch einen höhern göttlichen Beystand überwinden können“. ${ }^{21}$ Damit ist das Ziel klar umrissen, nicht jedoch der Weg. Beschritten werden aber kann er jedenfalls von jedem, „die Güter des Herzens bieten sich allen Sterblichen an“; gebahnt wird er durch „Gesetze der Vernunft und des Gewissens ${ }^{\star 22}$ :

Erwirb dir, so lehret die Vernunft und die Erfahrung, erwirb dir eine deutliche, überzeugende und vollständige Erkenntniß deiner Pflichten, ihrer Nothwendigkeit und Vortrefflichkeit; erneure und befestige diese Erkenntniß oft, bewahre sie vor Irrthümern, und wende sie sorgfältig auf das Leben und die Ausübung an, und lerne es empfinden, daß deine Pflicht, auch die schwerste, dein Glück ist. $^{23}$

Das erfordert zuallererst eine rigide Affektkontrolle; die Triebnatur des Menschen wird zwar nicht negiert, aber sie wird an eine - kurze - Leine genommen:

18 Bernd Witte: Der Roman als moralische Anstalt. Gellerts 'Leben der schwedischen Gräfin von G...' und die Literatur des achtzehnten Jahrhunderts, in: GRM 61, 1980, S. 150-168.

19 Christian Fürchtegott Gellert: Moralische Vorlesungen, in: ders.: Gesammelte Schriften. Kritische, kommentierte Ausgabe, Bd. 6, hg. v. Sibylle Späth, Berlin/New York 1992, S. 1-285; hier: Erste Vorlesung, S. 13.

20 Ebd., S. 19.

21 Ebd., S. 21.

22 Ebd., S. 19.

23 Ebd., S. 21. 
Wache über deine Leidenschaften und deine Sinnlichkeit, sie verführen dich; setze daher ein weises Mißtrauen in dich selbst, und prüfe täglich dein Herz und deinen Wandel mit Aufrichtigkeit [...]. ${ }^{24}$

Diesseitiger Effekt und Teilziel ist eine ideale Kommunikationsgemeinschaft: Eine allgemein waltende „Wahrheit des Herzens“, rückhaltlos ehrliche Kommunikation, resultiert in „Geselligkeit und Freundschaft“ und läßt zuletzt die Hypothese sich selbst erfüllen, daß „die ganze Welt [... ] eine große Familie [sei], die von dem weisesten, und mächtigsten, und gütigsten Wesen regiert wird, das über alle wacht, und dessen Liebe unendlich ist“ ${ }^{25}$

Auch Gellert aber ist klar, daß die Verwirklichung der Idee einer idealen Kommunikation eine Reihe von praktischen Problemen aufwirft - das wird ihm nicht zuletzt seine Praxis als Hochschullehrer vor Augen geführt haben. Das größte Problem ist die geforderte Differenzierung zwischen Oberfläche und Tiefe, zwischen Äußerung und Intention oder allgemein zwischen Schein und Sein: „Wir unterscheiden selten das, was der Mensch wirklich ist, von dem, was er zu seyn scheint, und zu scheinen sich bemüht.“ Das beobachtbar Prekäre der Differenzierung hat seinen natürlichen Ort: die „so genannte große Welt“. Sie werfe ein „falsches Licht“ auf den Menschen und erzeuge so aus dem (schönen) Schein Vorstellungen eines Seins, aus denen sich „mannichfaltige Irrthümer und Blendwerke der Einbildung“ herleiten; diese stärken die Tendenzen falschen Seins im richtigen, weil sie „den betrügerischen Begierden, die schon in uns da sind, gleichsam das Leben ertheilen, und uns zu einer thörichten Nachahmung andrer Menschen verführen“. ${ }^{26}$

Damit wird der höfischen Gesellschaft und ihrer Kommunikations- und Handlungsstruktur eine klare Absage erteilt. Wie sich aus Schein und Verstellung Machtzugewinn ableiten läßt, das zeigen die Lehren der politischen (Privat)Klugheit. ${ }^{27}$ Gellert nun versucht, den Klugheitsbegriff für sein bürgerliches Idealmodell zu retten, indem er ihn von den Zumutungen der Politik entkleidet, indem er politische Klugheit als falsche, auf den Schein abzielende Lehre entlarvt.

\footnotetext{
24 Ebd. - Zum Herzen als Instanz einer aufgeklärten Sinnlichkeit vgl. Sibylle Späth: Vom beschwerlichen Weg zur Glückseligkeit des Menschengeschlechts. Gellerts Moralische Vorlesungen und die Widerstände der Realität gegen die empfindsame Gesellschaftsutopie, in: „Ein Lehrer der ganzen Nation“. Leben und Werk Christian Fürchtegott Gellerts, hg. v. Bernd Witte, München 1990, S. 151-171; hier: S. 158.

25 Erste Vorlesung [Anm. 19], S. 20.

26 Achte Vorlesung [Anm. 19], S. 98.

27 Vgl. Ursula Geitner: Die Sprache der Verstellung. Studien zum rhetorischen und anthropologischen Wissen im 17. und 18. Jahrhundert, Tübingen 1992 (Communicatio, Bd. 1), S. 67 ff.; Georg Stanitzek: Blödigkeit. Beschreibungen des Individuums im 18. Jahrhundert, Tübingen 1989 (Hermaea. Germanistische Forschungen, N.F. Bd. 60), S. 40 ff.
} 
Sie nämlich entspringe derselben übermäßigen Selbstliebe, die sie dann rückkoppelnd stärke: „Der Mensch“ will

in Andern Achtung und Bewunderung erwecken, weil er stolz ist, weil dieser Stolz seiner Einbildung schmeichelt, weil Achtung und Bewunderung ihm Unterwürfige, Dienstfertige und Sklaven seiner Leidenschaften verschaffen. Was diese Absichten befördert, hält er für Klugheit; und diese Klugheit ahmen wir blindlings nach.

Seelenheil, ob jenseitiges, ewiges oder diesseitiges, vorläufiges, ist auf diesem Wege nicht zu erreichen. Kategorisch erklärt Gellert, „daß das Kleid, der Aufzug, das Gefolge, der Stand, das Geschlecht, die Miene, das Gespräch, die äußere Lebensart“ auf keinen Fall das „wahre Glück“ des Menschen bedeuten. ${ }^{28}$

Damit ist aber das Programm, wenn nicht des gesamten Romans über das Leben der Schwedischen Gräfinn von $G^{* * *}$, so doch zumindest das der Erziehung der Gräfin umrissen, wie sie es zu Beginn ihrer Erzählung selbst vorstellt:

Mit einem Worte, mein Vetter lehrte mich nicht die Weisheit, mit der wir in Gesellschaft prahlen, oder wenn es hochkömmt, unsere Ehrbegierde einige Zeit stillen, sondern die von dem Verstande in das Herz dringt, und uns gesittet, liebreich, großmüthig, gelassen, und im stillen ruhig macht. ${ }^{29}$

Wie in den Vorlesungen ist es auch im Roman die „große Welt“ des Adels, der nicht nur das Gegenmodell des Falschen darstellt, sondern auch auf eine Weise agiert, die es erschwert, nach richtigen Prinzipien zu handeln. Die Intrigen des Prinzen sind nicht nur konventionalisiertes Ingrediens, Topos innerhalb der Romantradition $^{30}$, die Gellert für seine Zwecke fungibel macht; sie dienen nicht nur als Movens der Handlung, indem sie den Ehemann (vorerst) beseitigen, um die tugendsame Gräfin ungestört den lüsternen Zudringlichkeiten des Intriganten aussetzen zu können; mit ihrem leicht als moralisch verfehlt zu etikettierenden Charakter dienen sie als Modell einer falschen Wirklichkeit, als Ausdruck einer ritualisierten Kommunikation, deren konventionalisierte Oberfläche die darunterliegenden Leidenschaften kaum zu bemänteln vermag.

Überhaupt wird höfische Kommunikation, wird Oberschichten-Interaktion ganz im Sinne des Bildes gezeichnet, das die Moralischen Vorlesungen von der „so genannten großen Welt“ entworfen haben ${ }^{31}$ : „,kaum bey Hofe angekommen“,

\footnotetext{
28 Achte Vorlesung [Anm. 19], S. 98.

29 Christian Fürchtegott Gellert: Leben der Schwedischen Gräfinn von G***, in: ders.: Gesammelte Schriften. Kritische, kommentierte Ausgabe, Bd. 4, hg. v. Bernd Witte u. Werner Jung, Elke Kasper, John F. Reynolds, Sibylle Späth, Berlin, New York 1989, S. 1-96; hier: S. 4. 30 Vgl. Meyer-Krentler, Der andere Roman [Anm. 17], S. 89 ff.

31 Zur Hofkritik in der Schwedischen Gräfinn vgl. Bernd Heidenreich: Sophie von La Roche eine Werkbiographie, Frankfurt a.M./Bern/New York 1986 (Frankfurter Hochschulschriften zur Sprachtheorie und Literaturästhetik, Bd. 5), S. 49 ff.; vgl. auch Peter Uwe Hohendahl: Empfindsamkeit und gesellschaftliches Bewußtsein. Zur Soziologie des empfindsamen Romans am Beispiel
} 
wird die tugendsame Gräfin zum Objekt der „Menge der Aufwartungen“ und „Schmeicheleyen“, und sie hat, um nicht „eitel und hochmüthig“ zu werden, die „ganze Philosophie nöthig“, die ihr Vetter, ihr Gemahl und ihr Vater - sämtlich also diskursive Sachwalter Gellerts und seiner Moraldoktrin - ihr mit auf den Weg gegeben haben. ${ }^{32}$ Auch der ehemalige Reisegefährte ihres Mannes, Herr R -, urteilt ganz im Sinne seines Schöpfers, wenn er Gesellschaften deswegen „nicht leiden“ kann, weil in ihnen „so viel Zwang, so viel unnatürliche Höflichkeiten und so viel Verhinderungen, frey und vernünftig zu handeln“, anzutreffen sind. ${ }^{33}$ Die Fronten verlaufen eindeutig und scharf: hier Vernunft, Freiheit, aufgeklärtes Bürgertum, dort Unnatur, Zwang, bienséance, decorum, unaufgeklärter Absolutismus. Galanterie ist bloß ein anderer Name der Lüge: Zu meiden ist - im Sinne der Abwehr „thörichter Nachahmung“ - der Umgang mit

solche[n] Mannspersonen, die insgemein für galant ausgeschryen werden, und die sich bemühen, ein junges Mädchen durch niederträchtige Schmeicheleyen zu vergöttern; die ihr durch ieden Blick, durch iede Bewegung des Mundes und der Hand von nichts als einer abgeschmackten Liebe sagen. Solche Leute müssen freylich nicht die Sittenlehrer der Frauenzimmer werden, wenn man haben will, daß eine junge Schöne keine Närrin werden soll. ${ }^{34}$

Das Gegenmodell einer aufrichtigen Kommunikation trägt das Etikett „zärtlich““ ${ }^{35}$ Charakteristisch ist, daß sie sich nicht innerhalb des Herrschaftsbereichs höfischer Etikette, sondern nur im als außergesellschaftlich gedachten Bereich, im Refugium des „Landguts“, installieren und aufrechterhalten läßt - nur hier läßt sich „ruhig und zärtlich“ leben. ${ }^{36}$ Für ein solches Leben qualifiziert hat sich der Graf bereits in seinem werbenden Brief gezeigt, der die entscheidenden Schlüsselbegriffe und positiven Reizworte als Eckpunkte seines Wertesystems anführt: „Zärtlichkeit“, „Freundschaft““ und deren exklusiv-intime Ausprägung: „Liebe““ ${ }^{37}$

Mit den Möglichkeiten, bloße empfindsame Rhetorik von echter Tugend zu unterscheiden, ist es nicht allzu weit her; es gibt schlechterdings keine Kriterien, die es erlaubten, unter eine 'gute' Oberfläche angepaßter Kommunikationsformen zu schauen und zu prüfen, ob ihnen eine 'gute' Eigentlichkeit zu Grunde liegt. Das zeigt Gellerts Roman ausführlich in einer der wichtigeren unter den vielen

von 'La Vie de Marianne’, 'Clarissa', ‘Fräulein von Sternheim’ und 'Werther’, in: Jahrbuch der Deutschen Schillergesellschaft 16, 1972, S. 176-207; hier: S. 197; ders: Der europäische Roman der Empfindsamkeit, Wiesbaden 1977 (Athenaion Studientexte, Bd. 1), S. 77 ff.

32 Leben der Schwedischen Gräfinn von G*** [Anm. 29], S. 13.

33 Ebd., S. 14.

34 Ebd., S. 37.

35 Zur „Ausdifferenzierung des Empfindsamkeitsdiskurses unter dem Schlagwort der Zärtlichkeit“ vgl. Wegmann, Diskurse der Empfindsamkeit [Anm. 4], S. 40 ff.

36 Leben der Schwedischen Gräfinn von G*** [Anm. 29], S. 14.

37 Ebd., S. 5f. 
Episoden, in denen die Vorsehung die constantia der Heldin, ihre „Gelassenheit und Geduld im Unglücke، ${ }^{38}$, versucht - freilich, ohne daß er die Konsequenzen für sein Modell diskutierte. (Wie alle Episoden dient auch diese letztlich bloß dazu, daß die in ihrem Verlauf gefährdete Ruhe am Ende wiedergewonnen erscheint.)

Nach dem Tode Carlsons, der die inzestuöse Ehe mit Mariane aufs eleganteste auflöst, lebt die empfindsame Gemeinschaft „wieder ruhig“, als „mitten in [der] Zufriedenheit“ Herr Dormund, „Carlsons guter Freund“, auftritt. Als Emblem seiner Freundschaft mit dem Verstorbenen überbringt er die Uhr mit Marianes Porträt. Er wird sofort in die Gemeinschaft aufgenommen, denn Freundschaft gilt als transitive Relation: „was brauchte er zu seiner Empfehlung mehr, als den Namen eines guten Freundes von unserm Carlson?“39 Der Augenschein moralischer Oberflächenqualitäten dient dann bloß zur Verstärkung eines fast a priori getroffenen moralischen Werturteils, denn obendrein ist Dormund „,von Person sehr angenehm“ - kurz: „Er gewann unsere Vertraulichkeit sehr bald.“ Auch seine diskursive Performanz gibt zu keinerlei Zweifel Anlaß; daß er, der nicht studiert hat, „etlichen Büchern und dem Umgange“ nur „einen gewissen Witz“ zu danken hat und zudem nur „,im Anfange“ damit zu überzeugen wußte ${ }^{40}$, sind offenbar von der Gräfin in der Erzählung nachträglich vorgenommene Relativierungen und Temporalisierungen. Zunächst nämlich erhält Dormund die von ihm überbrachte Uhr, und „auf die Uebergabe der Uhr folgt[] bald die Uebergabe des Herzens“ durch Mariane. $^{41}$

Daß überhaupt ans Licht kommt, wie sehr zu Unrecht Dormund das Vertrauen aller genießt, verdankt sich dem Umkehrschluß aus einem Prinzip, das der Roman extensiv vorführt. Schon der alte Graf, der Schwiegervater der Protagonistin, gibt auf dem Sterbebett explizit ein Beispiel, „wie leicht und glückselig man stirbt,

\footnotetext{
38 Diese zählen Gellerts Moralische Vorlesungen neben „Mäßigung und Beherrschung [der] Begierden“, „Ehrfurcht und Liebe gegen Gott“ und einigen anderen zu den „vornehmsten [...] Tugenden“ und „letzten und höchsten Güter[n] des Menschen“ (Erste Vorlesung [Anm. 19], S. 17f.); vgl. auch Späth, Vom beschwerlichen Weg [Anm. 24], S. 159f. - Das Motiv der immer wieder sich bewährenden constantia ist so aufdringlich, daß Carsten Schlingmanns Versuch einer Revision des Gellert-Bildes wohl doch etwas zu weit ausgreift, wenn er bloß zugestehen will, „daß Gellert wenigstens hier und da Reflexionen einstreuen mußte, um sich nicht dem Vorwurf auszusetzen, er habe ein gänzlich nutzloses Buch verfaßt“. Wenn sich an Gellerts Roman eine „elementare Lust“ ablesen läßt, dann aber wohl weniger eine „am Fabulieren“ als vielmehr an der moralischen Erbauung. An der Plausibilität von Schlingmanns Zurückweisung der These, Gellert sei als Romanautor im wesentlichen (Richardson-)Adept geblieben, ändert dies freilich nichts. Vgl. Carsten Schlingmann: Gellert. Eine literarhistorische Revision, Bad Homburg v. d. H. / Berlin / Zürich 1967 (Frankfurter Beiträge zur Germanistik, Bd. 3), S. 140 ff.; Zitate: S. 142.

39 Leben der Schwedischen Gräfinn von G*** [Anm. 29], S. 32f.

40 Ebd., S. 33.

41 Ebd.
} 
wenn man vernünftig und tugendhaft gelebt hat““. ${ }^{42}$ Diesem Beispiel werden noch Steelys Vater und der Graf, der Ehemann der Protagonistin, folgen. Der Umkehrschluß („Man nehme nur den Unglücklichen die Hoffnung einer bessern Welt: so sehe ich nicht, womit sie sich aufrichten sollen““43) führt zuletzt dazu, daß fehlende immanente Kriterien für Intentionen handelnder Personen durch transzendente Eingriffe ersetzt werden. Das aber setzt voraus, daß angesichts seines Todes vom Sünder immerhin soviel Tugend aufgebracht wird, daß er die Folgen seiner Untugend fürchten lernt, daß er - so die erste der Moralischen Vorlesungen - „an die Bestrafungen des Lasters [...] auf eine ganze Ewigkeit hinaus“44 denkt. Das ist eine starke Hypothese, mit der nur moraltheoretisch, nicht jedoch (kommunikations-)praktisch etwas gewonnen ist: Der finale Punkt, an dem die Tugend endlich obsiegt, wird in der sündhaften Individualbiographie so weit zum Ende verschoben, daß die Annahme seiner Notwendigkeit nicht schon in offensichtlichem Widerspruch zum empirischen Befund einer (auch) von Unmoral bestimmten Welt steht; für die soziale Interaktion des moralischen Subjekts - und damit für die mit ihm in Gemeinschaft Lebenden - hat das aber schlicht keine Konsequenz, denn es betrifft nur die rückschauende moralische Selbstbewertung und - wenn die Hoffnungslosigkeit angesichts des Jenseits so drückend geworden ist, daß sie an die Oberfläche drängt - die moralische Bewertung durch die anderen.

Der Roman macht das Dilemma deutlich, ohne es auszusprechen. Die rudimentäre Reaktion auf Dormunds Beichte besteht bloß darin, daß der Graf, „alle seine Vernunft und Religion zu Hülfe“ nehmend, dem „Unglückseligen“ in seiner Verzweiflung beizustehen sucht, allein vergebens. ${ }^{45}$ Daß es gerade die Finalität, der erwartete Übergang in die Transzendenz ist, der zuletzt die Tugend triumphieren läßt, macht der Text fast überdeutlich. Der von allen - einschließlich seiner selbst - mit Gewißheit erwartete Tod Dormunds tritt zwar gar nicht ein, aber die Wiedergesundung wird zum blinden Motiv. Dormund ist moralisch annulliert und muß aus moraltheoretischen wie erzähltechnischen Gründen - weil es keinen Ausweg gibt aus der Zwickmühle zwischen Haß und Mitleid dem Mörder gegenüber ${ }^{46}$ - als Figur fallengelassen werden. Die erste Fassung des Romans nimmt die Figur noch deutlich und explizit aus dem Spiel, indem schon an dieser Stelle kein Zweifel daran gelassen wird, daß sie nicht wieder auftauchen

42 Ebd., S. 12.

43 Ebd., S. 36.

44 Erste Vorlesung [Anm. 19], S. 21.

45 Leben der Schwedischen Gräfinn von G*** [Anm. 29], S. 34.

46 Vgl. ebd., S. 35. 
wird: „Dormund war fort. Wir haben auch in unserm Leben nichts weiter von ihm gehört. “47 Die Fassung der zweiten Auflage (1750) dagegen versucht, das lose Ende des narrativen Strangs zu kaschieren, indem die (freilich nicht eintretende) Möglichkeit offengelassen wird, Dormund könnte erneut ins Spiel kommen; lapidar heißt es hier: „Dormund war fort, ohne daß wir wußten, wohin. ““8

Das Fräulein von Sternheim, dessen Geschichte 23 Jahre nach Gellerts Roman erschien, hat gegenüber dessen moralischer Heldin keinen Vorteil erlangt, was Kriterien zur Unterscheidung falscher von wahrer Empfindsamkeit angeht. Auch sie, die anders als die schwedische Gräfin bereits Gegenstand einer Intrige ist, bevor sie an den Hof gelangt - sie soll in der Hauptstadt D. dem Landesherrn als Mätresse zugeführt werden -, vermag dem höfischen Treiben nur vergleichsweise starre Tugend entgegenzusetzen; diese aber taugt nur zur Bewertung offenkundigen Fehlverhaltens (im Sinne der Beobachterin), nicht zur Analyse verdeckter unmoralischer Operationen, wie die weitere Folge der Handlung zeigt.

Der Hof erscheint der Protagonistin charakterisiert durch funktionalen Diskurs, durch „eine allgemeine Sprache, die der Geistlose eben so fertig zu reden weiß, als der Allervernünftigste“ “. ${ }^{49}$ An der Sprache des Hofes läßt sich keine eigentliche Semantik ablesen; zudem verstört, wovon geredet wird: „Hoferzählungen“ „Ehrgeiz und Liebes-Intriguen, Tadel, Satyren“. ${ }^{50}$ Das Fräulein vermag gerade einmal einen der zentralen Grundsätze politischer Klugheitslehren aus den höfischen Diskursen, genauer: aus der Inszenierung religiöser Oberflächenrhetorik bei gleichzeitiger Absehung von moralischem Gehalt, zu abstrahieren: „kein Laster darf ohne Maske erscheinen“. ${ }^{51}$ Mehr als die im Umkehrschluß deduzierte Generalhypothese, daß sich unter einer moralischen „Maske“ auch 'lasterhafte’ Absichten verbergen können, ist daraus nicht zu gewinnen. Einen vermeintlichen Ausweg bietet ein Grundsatz des „Papas“ der Sternheim. Da Gesinnungsethik den moralisch Urteilenden vor das Problem einer wenig Sicherheit versprechenden Hermeneutik der Intention stellt, scheint die Einführung von Erfolgsethik geboten, die „gute Handlungen viel ruhmwürdiger [...] als die feinsten Gedanken“ erscheinen läßt. ${ }^{52}$ Das aber können sich auch diejenigen zu Nutze machen, denen daran gelegen ist, sich „Masken“ für ihre Laster zu verschaffen.

47 Ebd.

48 Ebd., S. 235 (Kritischer Apparat).

49 [Sophie von La Roche:] Geschichte des Fräuleins von Sternheim [...], hg. v. C. M. Wieland. Nachdruck der Ausgabe von 1771, hg. v. Kuno Ridderhoff, Berlin 1907 (Deutsche Literaturdenkmale des 18. und 19. Jahrhunderts, Bd. 138), S. 59.

50 Ebd., S. 70.

51 Ebd., S. 100.

52 Ebd., S. 97. 
Die Untauglichkeit ihrer moralischen Hermeneutik läßt das Fräulein von Sternheim den Wunsch nach Rückzug in seinen „ruhigen Cirkel von Beschäfftigung“ fassen. Der aber ließe sich mit Erfolg nur antreten, wenn Sophie tatsächlich, wie sie meint, die „große Welt [...] in allen Theilen richtiger zu beurtheilen wüßte“53, wenn die Trennlinie zwischen Gesellschaft und Privatheit überhaupt korrekt gezogen werden könnte; dazu aber müßte der empfindsame Diskurs über Ausschlußstrategien verfügen - jedoch: Auch falsche Freunde erscheinen als Freunde.

Lord Derby, der Schurke im Spiel, hat seinem Opfer eines voraus: Er ist nicht das Subjekt einer notwendig auf der Oberfläche verharrenden Beobachtung, sondern deren Objekt. Da auch er aber die Kriterien, die sich nur an die moralischen Äußerungsformen anlegen lassen, kennt, kann er sie in tugendsamer Mimikry zu seinem Nutzen einsetzen. Dieses Spiel der „abscheulichen Ränke“654 gerät so erfolgreich, daß auch der geläufige Rückzug der Hermeneutik auf den common sense nicht verfängt: Derby spielt die Rolle des Tugendboldes so überzeugend, daß seine Performanz sogar angesichts des Sternheimschen Moralrigorismus zwingender ausfällt als das allgemein akzeptierte moralische Urteil über ihn (,alle halten ihn für einen bösen Menschen“), was Sophie dazu verleitet, doch den Schluß auf seine Intentionen zu ziehen:

Derby ist rasch und unbesonnen; aber voller Geist und Empfindsamkeit. Wie schnell wie eifrig thut er Gutes? Sein Herz kann nicht verdorben seyn, weil er so viele Aufmerksamkeit für gute Handlungen hat; ich möchte bald hinzusetzen, weil er mich und meine Denkungsart lieben kann. ${ }^{55}$

Da ausgerechnet er selbst sich als Ausweg aus allem Argwohn präsentiert, gelangt er an sein Ziel: völlige Verfügungsgewalt über Sophie von Sternheim und ihre Tugend. Für seine verkappte Entführung wird er - zunächst - weniger bestraft; vielmehr wirkt sich auch bei ihm das Grunddilemma der bürgerlichen Liebesheirat aus: die zweifelhafte Eignung der Ehe zu ihrem Zweck, Liebe auf Dauer zu stellen $^{56}$ - was Gellert im Dienste einer Propaganda zur Einführung der Institution ${ }^{57}$ noch als gelingend vorführen konnte.

Während für die schwedische Gräfin die - auch ihr mißlingende - Unterscheidung von Oberflächenrhetorik und Tiefenintention noch ein Randproblem innerhalb eines Programms vorgeführter constantia in den Wirren der Zeitläufte

53 Ebd., S. 172.

54 Ebd., S. 187.

55 Ebd., S. $167 f$.

56 Zur romantischen Liebe und Liebesheirat vgl. Niklas Luhmann: Liebe als Passion. Zur Codierung von Intimität, Frankfurt a.M. 1982, S. 178 u. pass.

57 Vgl. Witte, Der Roman als moralische Anstalt [Anm. 18], S. 161. 
darstellte, ist die Notwendigkeit dieser Differenzierung bei ihrer gleichzeitigen Unmöglichkeit in der Geschichte des Fräuleins von Sternheim zum Zentralproblem geworden. Und es liegt in der Natur der Sache, daß Sophies Frage an ihren Gott als Zurechnungspunkt aller moralischen Grundsätze eine rhetorische ist: „aber, o Gott! wo, wo soll ein Herz wie dieß, das du mir gabst, wo soll es den Gedanken hernehmen, bey einer edlen, bey einer guten Handlung böse Grundsätze zu argwohnen! ‘58

Gegenüber der Protagonistin des Romans hat dessen Leser einen entscheidenden Vorteil. Dieser verdankt sich der Tatsache, daß der für den moralischen Briefroman $^{59}$ konstitutive Grundsatz, „alles sagen“ zu müssen, auch für den Verführer gilt: „Weil man doch“, so Derby an „Milord B*“ in Paris, „,immer einen Vertrauten haben muß ${ }^{\text {“60 }}$ - das fordert die Textsorten-Taxonomie in fast höherem Maße als die bürgerliche Moral. Diesem Grundsatz folgend, lüftet auch der Bösewicht die Maske seiner Laster - für niemanden sichtbar als für seine Leser, innerhalb wie außerhalb des Textes.

Die vorgeführte Hermeneutik offen dargelegter, sonst verborgener Absichten verschleiert das grundsätzliche Problem bloß. Aber die Verschiebung der Lektürezugriffe von Absichten, die sich aus Handlungen wie aus Äußerungen nicht ablesen lassen, auf Absichten, die für eine buchstäbliche Lektüre von Absichten schon aufbereitet sind, läßt sich auch auf der Seite der moralisch Zweifelsfreien durchführen. Zur Konsolidierung dieser moralischen Hermeneutik, die zugleich über deren grundsätzliche Schwächen hinwegtäuschen soll, wird eine solche Lektüre im Text schon vorgeführt.

Wielands Vorwort fingiert ${ }^{61}$ etwas, was gemeinhin zu den wenigen eindeutigen Anlässen für Ausschluß aus dem empfindsamen Diskurs zählt: einen Vertrauensbruch; inszeniert er die Herausgabe des Textes seiner „Freundin“ Sophie von La Roche doch als „Verrätherey“, die zu rechtfertigen die prätendierte Intention seines Widmungstextes an die Autorin ist. Diese nämlich habe eigentlich auf eine

\footnotetext{
58 Geschichte des Fräuleins von Sternheim [Anm. 49], S. 215.

59 Zu dessen Poetik generell vgl. Wilhelm Voßkamp: Dialogische Vergegenwärtigung beim Schreiben und Lesen. Zur Poetik des Briefromans im 18. Jahrhundert, in: DVjs 45, 1971, S. 80 116.

60 Geschichte des Fräuleins von Sternheim [Anm. 49], S. 108.

61 Vgl. Barbara Becker-Cantarino: „Muse“ und „Kunstrichter“: Sophie La Roche und Wieland, in: MLN 99, 1984, S. 571-588; hier: S. 575 ff. - Zu den „Entstehungsbedingungen des Romans“ vgl. Margrit Langner: Sophie von La Roche - die empfindsame Realistin, Heidelberg 1995 (Reihe Siegen, Bd. 126), S. 22 ff.; zur Rolle Wielands vgl. auch: Gudrun Loster-Schneider: Sophie La Roche. Paradoxien weiblichen Schreibens im 18. Jahrhundert, Tübingen 1995 (Mannheimer Beiträge zur Sprach- und Literaturwissenschaft, Bd. 26), S. 67 ff.; Monika Nenon: Autorschaft und Frauenbildung. Das Beispiel Sophie von La Roche, Würzburg 1988 (Epistemata. Reihe Literaturwissenschaft, Bd. 31), S. 51 ff.
} 
geradezu paradigmatisch exklusive empfindsame Kommunikation abgezielt, als sie jenem „unter den Rosen der Freundschaft ein Werk [i]hrer Einbildungskraft und [i]hres Herzens an[vertraut]“ habe. Wieland zitiert aus einem vorgeblichen Begleitschreiben des Romanmanuskripts, das dieses zum Supplement eines empfindsamen Briefwechsels zwischen der Autorin, die keine Autorin sein wollte, und einem Herausgeber, der nicht veröffentlichen durfte, stilisiert:

'Ich sende es Ihnen, (schreiben Sie mir) damit Sie mir von meiner Art zu empfinden, von dem Gesichtspunct, woraus ich mir angewöhnt habe, die Gegenstände des menschlichen Lebens zu beurtheilen, von den Betrachtungen, welche sich in meiner Seele, wenn sie lebhaft gerührt ist, zu entwickeln pflegen, Ihre Meynung sagen [...]. ${ }^{62}$

Wieland fungiert in der realen Herausgeberfiktion also nicht als erster in der Reihe der „Kunstrichter“63 (deren Wohlwollen er den Text in der Folge allzu demütig anempfiehlt), sondern als moralischer Richter der Autorin selbst.

Das Prinzip der Offenlegung von Intentionen soll gut aufklärerisch im Dienste moralischer Perfektibilität universalisiert werden, da es dem Herausgeber geeignet scheint, „Weisheit und Tugend, - die einzigen großen Vorzüge der Menschheit, die einzigen Quellen einer wahren Glückseligkeit - unter ihrem Geschlechte, und selbst unter dem meinigen, zu befördern“ ${ }^{6}{ }^{64}$

Globale Moralität rechtfertigt so die lokale Amoralität des Vertrauensbruchs. Jene sei zuletzt erfordert, weil der Moralität die Forderung maximaler Reichweite inhärent sei: „Ist es nicht unsre Pflicht, in einem so weiten Umfang als möglich Gutes zu thun?،65

Der empfindsame Briefroman wird in die Schwundstufe eines empfindsamen Briefwechsels integriert: in einen einzigen Brief, der die Position des Briefpartners aus sich ablesen läßt. Daran, daß diese empfindsame Metakommunikation gelingt, wird kein Zweifel gelassen, resultiert sie doch

in der angenehmsten Uebereinstimmung ihrer [sc. der Sternheim] Grundsätze, ihrer Gesinnungen und ihrer Handlungen mit den besten Empfindungen und mit den lebhaftesten Ueberzeugungen meiner [sc. Wielands] Seele. ${ }^{66}$

Die Tugendkataloge, die Wieland aufstellt, um die Lektüre des nachfolgenden Romans in moralisch gute Bahnen zu lenken, und die auf produktive Weise die Differenz zwischen der Tugend der Protagonistin und ihrer Autorin - die Wieland

62 Geschichte des Fräuleins von Sternheim [Anm. 49], S. 1 (Vorwort).

63 Ebd., S. 7.

64 Ebd., S. 2.

65 Ebd., S. 4.

66 Ebd., S. 3. 
sowieso schlicht miteinander identifiziert ${ }^{67}$ - verwischen, lassen keinen Aspekt der Empfindsamkeit als Teilbewegung der Aufklärung aus:

diese ungeschminkte Aufrichtigkeit der Seele, diese sich immer gleiche Güte, dieses zarte Gefühl des Wahren und Schönen, diese aus einer innern Quelle stammende Ausübung jeder Tugend, diese ungeheuchelte Frömmigkeit [...], dieses zärtliche, mitleidsvolle, wohlthätige Herz, diese gesunde, unverfälschte Art von den Gegenständen des menschlichen Lebens und ihrem Werthe, von Glück, Ansehen und Vergnügen zu urtheilen $[\ldots]^{68}$

- und:

[d]ie naive Schönheit ihres Geistes, die Reinigkeit, die unbegrenzte Güte ihres Herzens, die Richtigkeit ihres Geschmacks, die Wahrheit ihrer Urtheile, die Scharfsinnigkeit ihrer Bemerkungen, die Lebhaftigkeit ihrer Einbildungskraft und die Harmonie ihres Ausdrucks mit ihrer eigenen Art zu empfinden und zu denken $[\ldots] .{ }^{69}$

Die Offenheit, die der empfindsame Diskurs unbedingt fordert, führt folgerichtig zu einer Ablehnung der Rhetorik als einer Kalkulation der sprachlichen Mittel ${ }^{70}$; dies gilt dann auch für den Roman, der - wie der empfindsame Brief - eigentlich „einer künstlichen Form [...] entbehren“ kann, weil er „innerliche und eigenthümliche Schönheiten für den Geist und das Herz hat“. ${ }^{71}$

Wielands Kalkül einer Kalküllosigkeit, die deswegen so konstruiert ist, weil sie als einzige Fiktion des Textes nicht literarisch fingiert (also dem Leser als Angebot zu dessen Selbsttäuschung vorgestellt), sondern kontrafaktisch behauptet ist, ist nicht aufgegangen; denn die kritische Rezeption des Romans hat dessen künstlerische - und das heißt (auch): rhetorische - Qualität gegen die von Wieland behauptete Unmittelbarkeit für die Autorin reklamiert.

Die Leiden des jungen Werthers ${ }^{72}$ ist kein empfindsamer Text - jedenfalls nicht in dem Sinne, daß er funktionierende Kommunikation über innere Zustände der Kommunizierenden vorführte, daß er eine Hermeneutik des 'Herzens' prozessierte oder gar beschriebe. Das beginnt damit, daß über die Rezeption von Werthers Briefen durch deren Empfänger Wilhelm wenig bekannt wird. Das

67 Zu dieser Identifikation generell vgl. das Kapitel „Fräulein von Sternheim contra Mme. de La Roche“ in: Silvia Bovenschen: Die imaginierte Weiblichkeit. Exemplarische Untersuchungen zu kulturgeschichtlichen und literarischen Präsentationsformen des Weiblichen, Frankfurt a.M. 1979, S. 190-200.

68 Geschichte des Fräuleins von Sternheim [Anm. 49], S. 3 (Vorwort).

69 Ebd., S. 8f.

70 Die Ablehnung von Rhetorik für Brief und Briefroman im Sinne der „Kunst einer gewollten Kunstlosigkeit“ geht dabei nicht zuletzt auf Gellerts Briefsteller zurück; vgl. Voßkamp, Dialogische Vergegenwärtigung [Anm. 59], S. 82 ff.; Zitat: S. 85.

71 Geschichte des Fräuleins von Sternheim [Anm. 49], S. 6 (Vorwort).

72 Aus der überbordenden Fülle der Forschungsliteratur sei - wegen des universalen Charakters nur hervorgehoben: Horst Flaschka: Goethes 'Werther'. Werkkontextuelle Deskription und Analyse, München 1987. 
Wenige allerdings reicht aus, um klarzumachen, daß Werther von niemandem so sehr verstanden wird wie von Wilhelm und daß auch dieses Verstehen von Werther (mutwillig) preisgegeben wird. Werther bemüht sich nicht um Verstehbarkeit. Die Sprache seiner Briefe ist gekennzeichnet durch Anakoluthe, Aposiopesen, Abbrüche. Beschreibungen, Darstellungen, Erläuterungen werden beendet, nicht wenn die Rezeption des Gemeinten weitgehend sichergestellt ist, sondern wenn die erforderte Produktionshaltung nicht mehr durchgehalten werden kann: „Ein unerträglicher Mensch hat mich unterbrochen. Meine Thränen sind getroknet. Ich bin zerstreut. Adieu Lieber.“73

Selbst mit Lotte kommuniziert Werther kaum. Das zeigt sich auch an einer notorischen Stelle, die immer wieder als Beleg für das genaue Gegenteil angeführt worden ist ${ }^{74}$ : Wenn Lotte nach von der Tanzgesellschaft überstandenem Gewitter mit Werther am Fenster stehend, über diesem einen „Strom von Empfindungen“ mit der einfachen „Loosung“ „- Klopstock!“ 'ausgießt” ${ }^{75}$, dann zeigt das weniger den Gleichklang der Herzen als vielmehr die Tatsache, daß beide ähnlich lesen: identifikatorisch. So wie Lotte am liebsten liest, wenn sie im Text „ihre Welt wieder finde[t] $]^{\text {“76, }}$, schätzt Werther an seinem Homer vor allem, daß er ihn nachspielen kann, daß er seine eigentümliche ${ }^{77}$ 'patriarchalische' Lesart des Textes inszenieren, in seine „Lebensart verweben“ kann:

Wenn ich so des Morgens mit Sonnenaufgange hinausgehe nach meinem Wahlheim, und dort im Wirthsgarten mir meine Zukkererbsen selbst pflükke, mich hinsezze, und sie abfädme und dazwischen lese in meinem Homer. [...] Da fühl ich so lebhaft, wie die herrlichen übermüthigen Freyer der Penelope Ochsen und Schweine schlachten, zerlegen und braten. Es ist nichts, das mich so mit einer stillen, wahren Empfindung ausfüllte, als die Züge patriarchalischen Lebens, die ich, Gott sey Dank, ohne Affektation in meine Lebensart verweben kann. $^{78}$

Werther geht es weniger um empfindsame Kommunikation als um körperliche Entgrenzung, er befindet sich weniger in Lacans 'Spiegelstadium, ${ }^{, 79}$ als vielmehr

73 Der Text wird zitiert nach der dtv-,,Bibliothek der Erstausgaben“: Johann Wolfgang Goethe: Die Leiden des jungen Werthers, hg. v. Joseph Kiermeier-Debre, München 1997, unter Angabe des Briefdatums und der Seitenzahl der Erstausgabe (Leipzig 1774) in eckigen Klammern; hier: S. 98 [29. Juli 1772; 145].

74 Vgl. besonders: Richard Alewyn: „Klopstock!“, in: Euphorion 73, 1979, S. 357-364, hier: S. 360f.

75 Die Leiden des jungen Werthers [Anm. 73], S. 34 [16. Juni 1771; 44].

76 Ebd., S. 28 [16. Juni 1771; 34].

77 Vgl. Carol E. W. Tobol, Ida H. Washington: Werther's Selective Reading of Homer, in: MLN 92, 1977, S. 596-601.

78 Die Leiden des jungen Werthers [Anm. 73], S. 36f. [21. Juni 1771; 47f.].

79 Vgl. Reinhart Meyer-Kalkus: Werthers Krankheit zum Tode. Pathologie und Familie in der Empfindsamkeit, in: Urszenen. Literaturwissenschaft als Diskursanalyse und Diskurskritik, hg. v. Friedrich A. Kittler u. Horst Turk, Frankfurt a.M. 1977, S. 76-138, hier: S. 90 ff. 
auf der Suche nach der verlorenen Mutter-Imago. ${ }^{80}$ Mit der 'symbolischen Ordnung' setzt er sich nicht anders auseinander als durch Flucht.

Die Affektkontrolle, die von aufklärerischer Empfindsamkeit als direkte Begleitung der Offenlegung von Affekten gefordert wurde, setzt Werther mutwillig aus; folgerichtig glaubt er gar nicht erst an die Mitteilbarkeit von Empfindungen, besonders nicht gegenüber dem Vertreter der Kontrollinstanzen, Albert:

Mein Freund rief ich aus, der Mensch ist Mensch, und das Bißgen Verstand das einer haben mag, kommt wenig oder nicht in Anschlag, wenn Leidenschaft wüthet, und die Gränzen der Menschheit einen drängen. Vielmehr - ein andermal, davon sagt ich, und grif nach meinem Hute. O mir war das Herz so voll Und wir giengen auseinander, ohne einander verstanden zu haben. Wie denn auf dieser Welt keiner leicht den andern versteht. ${ }^{81}$

Die Geschichte der Empfindsamkeit und des empfindsamen Romans ist (auch) die Geschichte des Verhältnisses von Individualität und Sozialität. Während der frühempfindsame Roman der mittleren Aufklärung (und hier ist Gellerts Roman paradigmatisch), den empfindsamen Diskurs initiierend und proklamierend, seinen Protagonisten Individualität nur in begrenztem Maße zugesteht - so weit nämlich, daß sie als feste Größen moralischer Zurechnung konturiert sind - und der 'hochempfindsame' Roman sich um eine Vermittlung von Individualität und Sozialität bemüht (was bei Sophie von La Roche faktisch bedeutet, daß die Protagonistin ihre höchste Individualität in höchster Sozialität gewinnt, indem sie die ihr ganz persönlich gemäßen sozialen Tugenden übt), erteilt Werther den Forderungen der Sozialität eine unbedingte Absage. Die Entwicklung der Empfindsamkeit ist in ihrer Selbstaufhebung an einen Endpunkt gekommen, indem das Subjekt sich bloß dem Subjekt offenbart - in derselben antirhetorischen Rhetorik, wie sie für die Offenheit dem Anderen gegenüber gefordert worden ist. Denn Werthers Radikalität beschränkt sich nicht darauf, gegen die und abseits der Gesellschaft intime Zweierkommunikationen aufzubauen - ob mit Lotte ${ }^{82}$ oder mit seinem Briefpartner Wilhelm -, sondern er ist noch radikaler: Er verzichtet darauf, mit jemand anderem zu kommunizieren als mit sich selbst. Seine Briefe teilen sich eigentlich ihm selbst mit, und keinesfalls geht es ihm darum - seine

\footnotetext{
80 David Wellbery hat darauf hingewiesen, daß Werther gerade keine „fixation and stabilization of the ego within the domain of representation“ findet, sondern die Überschreitung seiner eigenen körperlichen Grenzen in Richtung auf einen ,absolute body“ sucht: „Goethe’s text is oriented toward a corporeal morphism - the absolute body - that comes into being as the dissolution of this domain [of representation].“ (D. E. Wellbery: Morphisms of the Phantasmatic Body: Goethe’s 'The Sorrows of Young Werther', in: Body \& Text in the Eighteenth Century, hg. v. Veronica Kelly u. Dorothea von Mücke, Stanford 1994, S. 181-208, hier: S. 185f., S. 193f.; Zitate: S. 186.)

81 Die Leiden des jungen Werthers [Anm. 73], S. 63f. [12. August 1771; 89].

82 Vgl. dagegen Wegmann, Diskurse der Empfindsamkeit [Anm. 4], S. 111.
} 
abwehrende Rhetorik zeigt dies deutlich -, daß Wilhelm ihm seine Meinung darüber sagte, was sich in Werthers Seele entwickelt, „wenn sie lebhaft gerührt ist“ ${ }^{83}$

Werther gelingt es nicht, das 'Eigentliche' seiner Intentionen, seine innersten Beweggründe mitzuteilen. Es gelingt ihm nicht in seinen Briefen - weder Wilhelm noch dem Leser des Romans gegenüber -, und es gelingt ihm auch gegenüber Lotte nicht, die durchaus plausibel argwöhnt, daß es „nur die Unmöglichkeit [sie] zu besizzen [sei], die [ihm] diesen Wunsch so reizend macht“"84, was aber doch wohl nicht in Einklang zu bringen wäre mit der Forderung nach aufrichtiger Liebe um der Individualität der Geliebten willen. Man könnte also boshaft formulieren, Werther (und seinem Autor) wäre es bloß um eine empfindsam-leidenschaftliche Oberflächenrhetorik zu tun; boshaft aber wäre hieran nur das „bloß“. Es stellt sich nämlich die Frage, ob es dem empfindsamen Diskurs um etwas anderes gehen kann als um Oberflächenrhetorik und was diese Vermutung, sollte sie zutreffen, für seinen Status als Diskurs bedeutet.

Der empfindsame Diskurs, die empfindsame Kommunikationsdoktrin fordert selbst auf das entschiedenste die Entsprechung zwischen Äußerung und Intention, zwischen der Oberfläche (die dann eben gerade keine rhetorische sein darf) und einer ihr zugrunde liegenden Eigentlichkeit. Er fordert die „naive“ Rede, wie sie Sulzer in seiner Allgemeinen Theorie der Schönen Künste mit Wielands Worten definiert:

Die Rede soll eigentlich ein getreuer Ausdruck unserer Empfindungen und Gedanken seyn. Die ersten Menschen haben bey ihren Reden keinen andern Zwek haben können, als einander ihre Gedanken bekannt zu machen, und wenn sie und ihre Kinder die angeschaffne Unschuld bewahret hätten, so wäre die Rede nach ihrer wahren Bestimmung ein offenherziges Bild dessen, was in eines jeden Herzen vorgegangen wäre und ein Mittel gewesen, Freundschaft und Zärtlichkeit unter den Menschen zu unterhalten. Jedermann weiß, daß die Sprache von den itzigen Menschen meistentheils gebraucht wird, andern zu sagen, was sie nicht denken noch empfinden, so daß die Rede demnach sehr selten ein Zeichen ihrer Gedanken ist. ${ }^{85}$

Im Verlauf der Entwicklung des Versuchs, eine 'Naivetät' der Sprache wiederzugewinnen, das Gesagte und das wirklich Empfundene zur Übereinstimmung zu bringen, gelingt es nicht, äußere Kriterien dafür anzugeben, wann eine solche Übereinstimmung besteht und wann nicht. Die Romane führen dies entweder vor, ohne daraus Folgerungen ableiten zu können (so etwa Gellert in der Dormund-

83 Geschichte des Fräuleins von Sternheim [Anm. 49], S. 1 (Vorwort).

84 Die Leiden des jungen Werthers [Anm. 73], S. 124 [Herausgeberbericht; 183].

85 Johann Georg Sulzer: Allgemeine Theorie der schönen Künste [...], 2. Teil, Leipzig 1775,

S. 297 (s.v. „Naiv“); zit. nach: Sauder, Empfindsamkeit [Anm. 2], Bd. I, S. 160. 
Episode), oder sie verschieben das Problem so, daß zumindest für den Leser alle Intentionen offen zu Tage liegen, weil auch und gerade die Bösewichte sich ihren Vertrauten gegenüber in schönster Offenheit äußern (so Derby in La Roches Roman). Der einzig produktive Ausweg scheint die effektvolle Inszenierung der Oberfläche bei gleichzeitigem Verzicht auf Offenlegung des Eigentlichen zu sein, das nicht zuletzt deswegen wohl in der Rezeption ein solches Gewicht gewonnen hat.

Der andere Ausweg, der vom Werther in viel geringerem Maß beschritten wird als von seinen Nachfolgern, ist der Versuch, Innerlichkeit non-verbal zu kodieren. ${ }^{86}$ Im Werther fungiert in diesem Sinne die große Zahl von Gedankenstrichen $^{87}$, die aber Unbestimmtheitsstellen bezeichnen, in die jeder Leser (und unter ihnen als erster Wilhelm) seinen eigenen Sinn hineinlesen kann. Am Ende dieses Entwicklungsstrangs steht Millers Siegwart, in dem kaum noch wirklich kommuniziert, dafür aber umso mehr geweint wird.

Das Problem, daß sich aus Äußerungen Intentionen nicht zweifelsfrei ablesen lassen - weil es nämlich die 'politische’ Kunst der Verstellung gibt -, wird von der rationalistischen Seite der Aufklärung erheblich grundsätzlicher bedacht, die dafür das Programm der 'Kardiognostik’ entwirft, dem etwa Christian Thomasius und Johann Kaspar Lavater mit seiner Physiognomik zuarbeiten. ${ }^{88}$

Daß sich das, worüber als 'Empfindsamkeit' zu reden sich eingebürgert hat, in Foucaults Sinne sehr differenziert als „Formationssystem“ von Aussagen, als Diskurs mithin, beschreiben läßt, darüber kann kein Zweifel bestehen. Sich selbst zu formieren und zu organisieren aber vermochte dieser Diskurs nicht. Es ist nicht unwesentlich, daß Foucault für die Analyse von Diskursen das Prinzip der „Äußerlichkeit“ fordert: „Man muß nicht vom Diskurs in seinen inneren und verborgenen Kern eindringen, in die Mitte seines Denkens oder einer Bedeutung, die sich in ihm manifestieren. ${ }^{\text {(89 }}$ Der Diskursanalyse geht es also nicht - wie etwa der 'Geistesgeschichte' -

um die Rekonstruktion eines anderen Diskurses, um das Wiederfinden des stummen, murmelnden, unerschöpflichen Sprechens, das von innen die Stimme belebt, die man hört, um die Wiederherstellung des kleinen und unsichtbaren Textes, der den Zwischenraum der geschriebenen Zeilen durchläuft und sie manchmal umstößt. ${ }^{90}$

\footnotetext{
86 Vgl. auch Wegmann, Diskurse der Empfindsamkeit [Anm. 4], S. 81ff.

87 Vgl. Jürgen Stenzel: Zeichensetzung. Stilanalysen an deutscher Prosadichtung, Göttingen 1970 (Palaestra, Bd. 241), S. 40 ff.

88 Vgl. Geitner, Die Sprache der Verstellung [Anm. 27], S. 124 ff., S. 239 ff.

89 Foucault, Die Ordnung des Diskurses [Anm. 11], S. 37.

90 Foucault, Archäologie des Wissens [Anm. 12], S. $42 \mathrm{f}$.
} 
Für die Diskursanalyse ist der „Sinn einer Aussage“

nicht definiert durch den Schatz der in ihr enthaltenen Intentionen, durch die sie zugleich enthüllt und zurückgehalten wird, sondern durch die Differenz, die sie an andere, wirkliche und mögliche, gleichzeitige oder in der Zeit entgegengesetzte Aussagen anfügt. ${ }^{91}$

Wenn etwas als Diskurs aufgefaßt wird, interessiert nicht, was von seinen (geäußerten) Aussagen ‘eigentlich’ gemeint ist, es interessieren die „systematische Gestalt‘`92, die „Regelhaftigkeit“ des Diskurses sowie „seine äußeren Möglichkeitsbedingungen“.93 Dies ist deshalb wesentlich, weil Diskurse nicht nur rezeptiv - Aussageformationen sind; sie sind auch produktiv nichts anderes als Formen, die Produktion von Aussagen zu reglementieren, zu bestimmen, welche Aussagen zu ihnen gehören und welche nicht. Wissenschaften etwa - die wohl erfolgreichsten und konsistentesten Diskursformen - können sich gar nicht dafür interessieren, was Wissenschaftler eigentlich sagen wollen, sondern nur dafür, ob sie regelhafte, 'wohlgeformte' Aussagen produzieren. Mathematische Aussagen sind nicht 'wahr', weil sie zu sagen scheinen, was sie wirklich meinen, sondern weil sie sich formal beweisen lassen und folglich nicht in Widerspruch zu anderen mit 'wahr' bewerteten Aussagen stehen. Jemand ist Wissenschaftler, wenn sich seinen Äußerungen nicht ablesen läßt, daß er keiner ist.

Ein Diskurs aber, der kaum etwas anderes fordert als die Übereinstimmung seiner Aussagen mit ihrem „inneren und verborgenen Kern“, ist ein echtes Paradoxon. Die Empfindsamkeit versucht nicht nur, ein Formationssystem von Aussagen zu sein, Äußerungen als zum Diskurs gehörig zu bestimmen, sondern auch, Aussagen auf ihre verborgenen Kerne hin zu befragen, diskursive Äußerungen und subdiskursive Bedeutungen abzugleichen. Dafür kann es keine Strategien geben, denn die Wahrhaftigkeit auch der ausführlichsten Introspektion ist niemandem zugänglich als dem Subjekt (und Objekt) der Introspektion selbst.

Das organisierende Gemeinsame empfindsamer Texte, dasjenige, was sie rezeptiv als Aussagen eines Diskurses erscheinen läßt, ist das vergebliche Bemühen, mehr zu generieren als einen Diskurs, mehr zu regeln als die Oberfläche der Äußerungen. Der empfindsame Diskurs (im schwachen Sinne) läßt sich als ein Diskurs beschreiben, der seinen Diskurscharakter selbst nicht reflektiert, ihn nicht reflektieren kann, weil er seinem Wesen nach - oder nach dem, was er für sein Wesen hält - kein Diskurs (im starken Sinne) sein kann.

91 Michel Foucault: Die Geburt der Klinik, München 1973, S. 15.

92 Ebd.

93 Foucault, Die Ordnung des Diskurses [Anm. 11], S. 37. 